\title{
El papel de la mediación comunicativa entre la polarización y la democracia en Colombia
}

The role of communicative mediation between polarization and democracy in Colombia

\author{
Oscar Iván Londoño \\ Pardo \\ Universidad Externado de Colom- \\ bia \\ Orcid https://orcid.org/oooo- \\ ooo2-9307-5559 \\ oscarivanlondono- \\ pardo@gmail.com
}

\author{
Felipe Arturo Mora \\ Morales \\ Universidad Externado de Colom- \\ bia \\ Orcid https://orcid.org/oooo- \\ 0003-3172-0580 \\ femora98@gmail.com
}

\author{
Clara Inés Torres \\ Malaver \\ Universidad Externado de Colom- \\ bia \\ Orcid https://orcid.org/oooo- \\ 0002-4895-0190 \\ clara.torresm93@gmail.com
}

Resumen: En Colombia existe un clima de opinión donde la polarización, fruto de la coyuntura, se considera un tema de primer orden en la agenda política nacional. El presente estudio analiza el papel mediador de la comunicación entre la polarización y la estructura de las redes sociales en el espacio digital. A partir de un análisis cuantitativo de los datos obtenidos de la encuesta sobre comunicación política adelantada en Colombia durante el 2018, se concluye que las expresiones políticas online como variable mediadora, aumenta el tamaño de las redes sociales en plataformas digitales de personas con mayores niveles de polarización. Este estudio aporta a la discusión sobre el rol de la comunicación política en la configuración de escenarios de deliberación política en la democracia.

Palabras clave: Polarización ideológica, expresiones políticas online, redes sociales, democracia, tamaño de las redes sociales

\begin{abstract}
In Colombia, there currently exists a climate of opinion where polarization is considered a topic of first order in the national political agenda. This study analyzes the mediating role of communication in between polarization and the structure of social networks in digital spaces. Through a quantitative analysis of the data obtained by a survey advanced in Colombia during 2018 regarding topics of political communication, this study concludes that political expressions online as mediating variables, increases the size of social networks in the digital platforms of people with high levels of polarization. This study contributes on the discussion about the role of political communication in the configuration of spaces for political deliberation in a democracy.
\end{abstract}

Keywords: Ideological polarization, political expressions online, social networks, democracy, social network size

Fecha de recepción: 12/12/2019

Fecha de aprobación 09/01/2020

Cómo citar este artículo / How to cite this paper: Londoño Pardo, O. I., Mora Morales, F. A., \& Torres Malaver, C. I., (2020). El papel de la mediación comunicativa entre la polarización y la democracia en Colombia. Revista de Comunicación Política, 2, 11-28. https://doi.org/10.29105/rcp2-1 


\section{Introducción}

"No más divisiones entre izquierda y derecha. Somos Colombia. No más divisiones entre socialistas y neoliberales. Somos Colombia. No más ISMOS. Somos Colombia” fueron las palabras pronunciadas por el presidente de Colombia Iván Duque (Presidencia de la República, 2018), durante el acto de posesión el 7 de agosto del 2018. Estas palabras dan cuenta de un clima de opinión donde la polarización, fruto de la coyuntura, se considera un tema de primer orden en la agenda política nacional (Dueñas, 2019).

Este fenómeno adquiere relevancia en la agenda pública de forma reciente, remontándose a la división provocada por el proceso de paz y cristalizada en los resultados del plebiscito. Por otra parte, el fin del conflicto con las FARC, abrió un nuevo espacio político para la izquierda, adquiriendo un mayor protagonismo en la contienda electoral del 2018, generando temor y señalamientos hacia las propuestas de esta corriente por parte de sus adversarios. A esto se suma la profundización de la crisis venezolana y la incorporación de la idea del "castrochavismo" en la deliberación política (Villalobos, 2017).

El sentido común indica que la identificación partidista o ideológica con los extremos del espectro político impide el diálogo, aumenta la agresividad y la adhesión dogmática (Yarce, 2018), apelando fundamentalmente a las emociones (Villalba, 2019). A esto se suma las implicaciones que tiene el fenómeno de la polarización sobre la economía, afectada por la desconfianza que genera en el consumidor (Revista Dinero, 2019). Aunque la polarización es considerada un fenómeno dado, algunas investigaciones como la realizada por la Universidad de los Andes (Revista Semana, 2019) o por Rojas (2018) plantean que esta idea corresponde más a una percepción que a una tendencia de identificación ideológica con los extremos por parte de la ciudadanía.

Son múltiples los interrogantes en torno a la división de la sociedad colombiana en la esfera política. Entre los aspectos difusos sobre la polarización se encuentran las diferencias y similitudes entre su manifestación ideológica, afectiva y percibida, sus efectos sobre el funcionamiento del régimen político y su relación con la acción comunicativa.

Hace falta ahondar en la investigación sobre la cuestión de la polarización para entender la relación entre algunas de sus manifestaciones y elementos constitutivos de la democracia tales como la expresión de ideas en la esfera pública y la configuración de las redes sociales en las plataformas digitales. Si bien algunas investigaciones analizan la existencia de la polarización política en la sociedad colombiana, no se ha contemplado el lugar que ocupa la comunicación en la relación entre las personas con diferentes ideologías y la apertura, tamaño y exposición en la construcción de redes sociales. Ante este escenario surge la pregunta ¿Cómo afecta la comunicación, a partir de las expresiones políticas online, sobre la relación entre la polarización ideológica y el tamaño de las redes sociales en el espacio digital en Colombia? 
Tomando como referencia el Modelo de Mediación Comunicativa (O-S-R-O-R) (Shah et al. 2017), se analiza si las expresiones políticas online como elemento mediador altera el impacto de la polarización sobre la configuración de las redes sociales en Colombia durante el 2018. El Modelo de Mediación Comunicativa resulta oportuno al considerar el consumo de noticias, la expresión de ideas o la conversación sobre asuntos políticos como factores que alteran la relación entre la disposiciones psicológicas y afectivas, el contexto estructural en el que se relacionan las personas y sus efectos sobre las actitudes y comportamientos (Shah et al. 2017).

El objetivo de la presente investigación es analizar la relación entre la polarización ideológica y el tamaño de la red a partir de la mediación de las expresiones políticas online. Para analizar la relación entre los conceptos de polarización ideológica, expresiones políticas online y tamaño de las redes sociales, se plantean tres partes en el desarrollo de la investigación. En primer lugar, se hace un balance sobre las diferentes aproximaciones académicas de la relación entre estos conceptos y su definición. En segundo lugar, se desarrolla un análisis cuantitativo a partir de los datos obtenidos en la encuesta de comunicación política - 2018 estableciendo relaciones entre los conceptos de polarización ideológica, tamaño de las redes sociales y expresiones políticas online. Por último, se concluye con algunas reflexiones sobre el papel de la comunicación como factor mediador entre la polarización y la deliberación política en la democracia.

\section{Aproximaciones al concepto de polarización ideológica}

De acuerdo con algunas investigaciones, la polarización se asocia al aumento de la distancia social entre colectividades, la intolerancia, la homofilia o la conformación de grupos homogéneos (McCoy et al., 2018). La polarización ideológica se ha estudiado a partir de las diferencias naturales y de expectativa que existen entre partidos políticos, candidatos y el electorado (Katsambekis \& Stavrakakis, 2013; McCoy et al., 2018). Adicionalmente, puede ser considerada como una forma de medición, en la que separa un público en dos bandos que se dividen entre izquierda y derecha, o liberal y conservador (McCoy et al., 2018; Poole \& Rosenthal, 2006). Sin embargo, este concepto no solamente mide las distancias ideológicas en un momento en particular, sino también el desplazamiento de grupos sociales (Garcia-Guadilla \& Mallen, 2016).

En una sociedad democrática, los individuos utilizan etiquetas ideológicas (izquierda/ derecha, liberal/conservador) no solo para organizar acciones y divulgar comunicaciones dentro del mismo círculo ideológico, sino también para identificar a sus contradictores políticos (Plata, 2016). $\mathrm{Al}$ diferenciar dos bandos con identidades e intereses mutuamente adversos, se genera una brecha entre estos polos opuestos (Lozada, 2014). 
Una manera distinta de entender la polarización ideológica es examinándola como un proceso. Diferentes estudios han definido este concepto como el proceso en donde la multitud de diferencias convencionales dentro de una sociedad, van paulatinamente alineándose dentro de una sola dimensión en la que las personas se diferencian en términos de "nosotros" y "ellos" (McCoy et al., 2018) o en donde emergen dos tipos de distribuciones de observación (Fiorina et al., 2005).

Existen diferentes maneras de medir la polarización. La polarización política usualmente es medida en términos de la distancia que existe dentro de partidos políticos, élites políticas o electores (McCoy et al., 2018), pero también se realiza esta medición al determinar la percepción de distancia ideológica entre partidos frente un escenario legislativo (Abramowitz, 2010) o posicionando partidos políticos en una escala temática de izquierda-derecha (Delli Carpini \& Keeter, 1993). En términos de polarización ideológica, usualmente se mide por medio del posicionamiento de los individuos en una escala izquierda-derecha (Campbell, 2016; Plata, 2016) o separando a estos a partir de asuntos políticos (Abramowitz, 2010).

Investigaciones como la realizada por Campbell (2016) utilizan preguntas de auto ubicación ideológica, las cuales resultan problemáticas debido al sesgo de escala (Lindh et al., 2019). Como afirma Saiegh (2014), los encuestados pueden presentar sesgos de percepción tales como la existencia de un múltiple significado para diferentes personas o una interpretación distinta de los intervalos de la escala a la luz de su percepción ideológica.

En Colombia, las investigaciones sobre la polarización se han ubicado en su dimensión ideológica (Plata, 2016) y percibida (Rojas, 2018), a partir de una mirada descriptiva tomando en cuenta variables espaciales y sociodemográficas. En la presente investigación, la medición de la polarización ideológica se plantea a partir de la ubicación dentro de la escala unidimensional liberal-conservador tomando en cuenta la opinión de las personas sobre diferentes temas específicos (Fiorina et al., 2005).

\section{Polarización ideológica y redes sociales}

En la literatura se ha establecido la estructura de las redes sociales como un factor con efectos significativos en la polarización, toda vez que tienden a funcionar como un entorno de información de los individuos donde hay lugar a la difusión de mensajes políticos. Una perspectiva plantea que la exposición a diferentes redes de comunicación puede tener efectos de despolarización. Se señala que la presencia de desacuerdos políticos estimula el pensamiento crítico o la deliberación, fomentando así la comprensión de otras opiniones (tolerancia política) y, en última instancia, induciendo el consenso

entre los ciudadanos a través de la modificación de sus opiniones por aquellas favorecidas por otros (Lee et al., 2014). 
Otra mirada distinta señala que las tecnologías digitales facilitan la conectividad entre individuos y por tanto la conformación de redes, sin embargo, las personas conforman dichas redes bajo un criterio de homogeneidad ideológica (Rojas et al., 2016, p. 27). Esta tendencia, enraizada en la teoría de la exposición selectiva (Klapper, 1960) es considerada por los investigadores Valenzuela y Rojas (2019) como una disrupción en los canales informativos que podrían causar un impacto perjudicial a la democracia. Los llamados "filtros burbuja" incrementan la polarización pues fortalecen ideologías preexistentes en lugar de fomentar nuevos conocimientos (Valenzuela \& Rojas, 2019).

Desde la perspectiva crítica sobre el funcionamiento de las redes sociales, estas son culpadas a menudo de exacerbar la polarización política al crear “cámaras de eco" que previenen a las personas de la exposición a información que contradice sus creencias preexistentes. En contraposición a la mirada anterior, para Lee et al. (2014) los individuos que se encuentran más polarizados tienden a buscar información poco amigable con sus creencias, ampliando así el tamaño de su red, pues consideran estar seguros de su capacidad para contrarrestar dicha información. Aquellos que se encuentran más expuestos al desacuerdo político y se expresan políticamente con mayor frecuencia, tienden a tener un amplio repertorio argumentativo, es decir, una amplia gama de argumentos en contra y a favor de su posición política.

\section{Expresiones políticas online y tamaño de las redes sociales}

Las redes de comunicación ciudadanas pueden caracterizarse a partir del tamaño, la diversidad en la composición y la fuerza de las conexiones entre los individuos (Gil de Zúñiga \& Valenzuela, 2011). El tamaño de la red consiste en la existencia de lazos fuertes y lazos débiles (Park, 2017), la fuerza de los lazos hace referencia a la frecuencia de comunicación entre un individuo y las personas con las que tiene diferentes grados de intimidad (Gil de Zúñiga \& Valenzuela, 2011). Eveland et al. (2013) consideran que las redes pequeñas pueden ser confiables, pero a su vez transportan información redundante, mientras que las redes grandes pueden comprender una combinación de lazos fuertes y débiles, estos últimos terminan proporcionando información única en comparación con los lazos fuertes (Park, 2017). Esta apreciación es compartida por otros investigadores, quienes señalan que, en una red amplia, los lazos débiles no sólo ofrecen el compromiso cívico, sino también una información heterogénea (Park, 2017).

Para Kavanaugh et al. (2005), a medida que se incrementa el número de redes interpersonales y los micro o macro puentes que estas generan, se incrementa el potencial de nueva información y oportunidades de discusión y expresión pública. Asimismo, se ha planteado que el tamaño de las redes sociales se encuentra estrechamente relacionado con la posibilidad de entablar discusiones interpersonales y tiende a aumentar la exposición a diversas personas y puntos de vista (Park, 2017), 
por lo cual a medida que el tamaño de las redes de discusiones incrementa, lo hace el número de lazos débiles dentro de estas (Gil de Zuñiga \& Valenzuela, 2011).

La discusión política tiene un rol de mediación en la relación entre el tamaño de las redes y la polarización, teniendo en cuenta que la simple exposición a diversos puntos de vista no significa necesariamente que esta información sea considerada para determinar las opiniones políticas de las personas. Así, mediante el proceso de expresión y las discusiones políticas las personas buscan dar sentido a las nuevas ideas encontradas dentro de su red social, estimulando el pensamiento reflexivo (Lee et al., 2014). Las conversaciones políticas contribuyen a que los ciudadanos se encuentren más informados, comprometidos y activos (Rojas et al., 2011). La frecuencia de intercambio de expresiones políticas lleva a que las personas procesen la información adquirida con mucho más cuidado, ya que se encuentran preparadas al recibir información que difiere de su punto de vista político (Lee et al., 2014).

La expresión política en redes sociales se entiende como la habilidad de expresarse públicamente sobre temas políticos (Barnidge et al., 2018), la transmisión de cualquier contenido original producido por el usuario (blog, publicaciones, fotos, videos, memes, etc.) (Ardèvol-Abreu et al., 2017) y/o la distribución o reproducción de material no original del usuario compartido en sus perfiles o canales personales (enlazar, comentar, resaltar, compartir, etc.) (Goode, 2009; Nip, 2006). Esta distinción permite realizar un enfoque en una parte de la estructura unificada del modelo de la mediación comunicativa (Shah et al., 2017).

Algunos estudios han encontrado una relación positiva entre el tamaño de las redes y las expresiones: entre más heterogéneas sean las redes sociales de un individuo, más proclive es para realizar expresiones políticas online (Barnidge et al., 2018). Existen tres razones para que haya lugar a esta relación positiva. Primero, las personas que son confrontadas con opiniones políticas desagradables toman una "acción correctiva", es decir, sienten la necesidad de dar respuesta a contenidos hostiles. Segundo, la expresión política puede en algunas ocasiones tomar la forma de intento de persuasión sobre otras personas, en otras palabras, se intenta cambiar la opinión de los demás a partir de expresiones desagradables. Tercero, existen procesos basados en la identidad, donde la expresión política tiende a ser una expresión representativa y se encuentra motivada por la creencia de que las identidades particulares no han sido representadas en el discurso público (Barnidge et al., 2018).

Estudiar la relación entre la polarización ideológica, las expresiones políticas online y el tamaño de las redes en el caso de Colombia, permite reconocer las posibilidades o no de un diálogo pluralista que favorezca un escenario de democracia deliberativa. Por ello, este trabajo investigativo sostiene como hipótesis que las personas que se ubican en un mayor nivel de polarización ideológica, presentan un mayor tamaño de sus redes al realizar expresiones políticas online. 


\section{Modelo de mediación comunicativa (O-S-R-O-R)}

La comunicación efectúa un proceso mediador entre las disposiciones contextuales e individuales, el consumo de medios y sus efectos cognitivos y conductuales en el ámbito político. De acuerdo con Shah et al. (2017), el consumo de noticias y la discusión ya sea en línea o no, canaliza las influencias demográficas, disposicionales y contextuales sobre la participación política. A estos factores se suma la expresión pública de opiniones, asumiendo que la producción de mensajes tiene un mayor impacto en los efectos de la comunicación como elemento catalizador en el cambio de comportamientos, creencias y actitudes.

De acuerdo con la perspectiva inicial de este modelo, las conversaciones políticas y el consumo de noticias alimentan la participación cívica y la acción colectiva. Sin embargo, a partir de una revisión crítica hecha por algunos de los principales promotores del modelo (Shah et al., 2017), en un entorno caracterizado por la configuración en red de la esfera pública, debido a la participación activa de las audiencias en la producción, se hace necesario profundizar en el estudio de otros efectos de la mediación comunicativa distintos a la participación política, algunos relacionados con el funcionamiento de la democracia como la desconfianza social, la erosión de la legitimidad de las instituciones políticas o la polarización (Shah et al., 2017).

\section{Las redes sociales y la democracia deliberativa}

Habermas plantea como condiciones inherentes de la democracia la posibilidad de la deliberación a partir de la existencia de una espacialidad que permita el encuentro de diferentes puntos de vista e intereses. El espacio público es un escenario que permite a los ciudadanos interactuar frecuentemente a partir de prácticas discursivas (Domínguez, 2013). En cuanto a la deliberación, esta ha sido comprendía como "un acto en el que los ciudadanos consideran hechos relevantes desde diferentes puntos de vista y dialogan con los demás para pensar críticamente sobre las distintas opciones que tiene un asunto público; así, se incrementan las perspectivas, comprensiones y opiniones sobre un tema específico" (Rengifo, 2019).

Como señala Oquendo (2005) la democracia desde la perspectiva deliberativa se encuentra asociada al uso de la razón como fundamento para la resolución de los problemas comunes:

La noción de democracia deliberativa tiene sus raíces en el ideal intuitivo de una asociación democrática en la que la justificación de los términos y las condiciones de la asociación se lleva a cabo por medio de la argumentación y el raciocinio público de los ciudadanos en condiciones de igualdad. Los ciudadanos en una comunidad de esta índole comparten un compromiso con la resolución de los problemas de decisión colectiva a través del raciocinio 
público y consideran sus instituciones básicas legítimas en la medida en que éstas establecen un esquema para la deliberación pública y libre. (p.3)

Adicionalmente, se considera que esta se refiere a una actitud dirigida a la cooperación social, es un intercambio de opiniones a través del cual se permite ser persuadido por razones relacionadas con los derechos de los otros y de sí mismo (Domínguez, 2013). A propósito del concepto de la democracia deliberativa y su relación con las redes sociales como espacio público, Alconchel (2014) afirma que:

gracias a las redes sociales sería posible introducir mejoras en los procedimientos para el debate político y la toma de decisiones. A este concepto se le unen reflexiones como las de Thompson (1998), que insiste en la necesidad de encontrar maneras de incrementar la participación democrática mediante la ampliación de los procesos deliberativos. Es decir, afirma que es posible resolver cuestiones de forma creativa y colaborativa en red. (p. 31)

Existen dos argumentos opuestos frente a las redes sociales y su papel en la democracia deliberativa: en primer lugar, se considera que el uso de internet va en detrimento de la democracia deliberativa al facilitar el incremento de la exposición selectiva. Las personas aprovechan el control que les permite esta plataforma para exponerse a información ideológicamente consonante y abolir la exposición de diferencias políticas. Por el contrario, el segundo argumento plantea que el uso de internet contribuye a la democracia al incrementar la exposición de las personas a diferencias políticas a través de la debilidad de los límites sociales y al enlace de divisiones geográficas (Brundidge, 2010).

En este trabajo investigativo se asume la pluralidad de los contactos que se desprende de las redes sociales y el tamaño de estas como elementos que amplían el horizonte deliberativo de la democracia.

\section{Metodología de la investigación}

Para este estudio se usó como fuente de información la Encuesta Comunicación Política - Colombia 2018 elaborada por la Universidad Externado de Colombia, la Universidad de Wisconsin y la empresa DeProyectos S. A. S, donde se consideró como universo la población urbana mayor de edad, residentes en las ciudades de Bogotá, Medellín, Cali, Barranquilla, Cartagena, Cúcuta, Pereira, Manizales, Pasto y Villavicencio.

El cuestionario se distribuyó con base al grado de dispersión tanto como por estrato socioeconómico, teniendo como resultado final 1118 personas entrevistadas. La operación de recolección a nivel nacional inició el 28 de junio y culminó el 29 de julio de 2018. Los datos fueron sistematizados y analizados mediante la herramienta estadística SPSS. 
Para analizar el comportamiento de las variables, se utilizó el promedio como medida de tendencia central con el propósito de identificar la ubicación de las respuestas de los encuestados en las variables de escala (polarización ideológica, expresiones políticas online y tamaño de la red). La relación entre las variables fue medida usando la correlación de Pearson (Hernández Sampieri et al., 2010). La influencia de las variables independientes (polarización ideológica y expresiones políticas online) sobre la variable dependiente (tamaño de las redes sociales) fue estimada mediante la aplicación de regresiones lineales simples que permitieran contrastar la influencia de estas con variables de control. Para testear la hipótesis de investigación, se empleó el método de regresión logística con la técnica de bootstrapping de Andrew F. Hayes mediante la herramienta PROCESS macro para el software SPSS. Como afirman Lee et al. (2014), "esta técnica testea directamente una relación mediadora, mientras que otros métodos lo testean indirectamente al observar cambios en las magnitudes de asociación entre variables de interés" (p. 10). El análisis de las variables mediadoras se realizó con un intervalo de confianza del 95\%, diez mil (10000) muestras bootstrapping y el modelo 4 de esta herramienta (Hayes, 2017).

\section{Medición de variables}

Polarización ideológica. Se utilizaron 12 preguntas con una escala de 10 puntos, desde la posición más liberal hasta la más conservadora de acuerdo con las opciones de respuesta. La escala de la variable está compuesta por valores absolutos. Para identificar la ubicación de los encuestados en la escala, se utilizó el promedio de las respuestas obtenidas en cada pregunta como medida de tendencia central. Tomando en cuenta otras investigaciones (Lee et al., 2014), la polarización ideológica fue medida por el valor doblado del promedio de las respuestas a las preguntas sobre los temas anteriormente mencionados $(M=1.92, D E=1.04$, rango $=1-5)$.

Expresiones políticas online. La pregunta y las afirmaciones del cuestionario que se utilizaron para la definición de la variable fueron "Queremos entender cómo utiliza Facebook y Twitter. En una escala del o al 5, donde o significa nunca y 5 frecuentemente, con qué frecuencia en Facebook y Twitter usted: (...)": expresa sus opiniones sobre temas de actualidad, comparte noticias de medios tradicionales, comparte noticias de medios no tradicionales, comparte noticias o información con la que está de acuerdo y comparte noticias o información con la que no está de acuerdo.

En la construcción de la variable se tuvieron en cuenta las respuestas relacionadas con las redes sociales de Facebook y Twitter debido a la presencia simultánea de lazos débiles y fuertes en comparación con otro tipo de redes sociales (Rojas, 2018). Con el propósito de reconocer el nivel de frecuencia de las expresiones políticas online en su conjunto, se calculó el promedio de las respuestas dadas por cada persona $(M=2.66, D E=1.73$, rango $=0-5)$.

Tamaño de las redes sociales. Retomando el criterio de selección aplicado en la variable "expresiones políticas online", para el análisis empírico sobre el tamaño de las redes sociales se escogieron las preguntas relacionadas con el número de contactos en las redes de Facebook y Twitter 
(número de contactos o amigos en Facebook y número de personas o entidades que sigue o lo siguen en Twitter). Al medir la correlación entre las respuestas sobre el número de contactos que sigue o lo siguen a cada uno de los encuestados en la red social de Twitter, $r(169)=.67, p<.01$, se encontró una relación estadísticamente significativa, permitiendo la selección de la pregunta sobre el número de contactos que siguen los encuestados como elemento representativo para la construcción de la variable. Posteriormente, se estimó la correlación entre el número de contactos en Facebook con el número de personas que siguen los encuestados en Twitter, $r(160)=.30, p<.01 \mathrm{Al}$ estimarse una correlación estadísticamente significativa, se calculó el promedio como forma de medición del tamaño de las redes sociales $(M=325 \cdot 56, D E=404 \cdot 79$, rango $=2-5000)$.

Tabla 1. Opciones de respuesta a preguntas sobre polarización ideológica.

\begin{tabular}{|c|c|}
\hline Frase 1 (liberal) & Frase 2 (conservadora) \\
\hline No imposición de creencias religiosas & Creencias religiosas como base para las leyes. \\
\hline Distribución equitativa de la riqueza. & Incentivos para la iniciativa individual. \\
\hline Defender las libertades civiles. & Mantener la ley y el orden. \\
\hline $\begin{array}{l}\text { Mejorar la asistencia social e incrementar impues- } \\
\text { tos. }\end{array}$ & Reducir impuestos y reducir asistencia social. \\
\hline $\begin{array}{l}\text { Hombres y mujeres proveen por igual el sustento } \\
\text { para la familia. }\end{array}$ & $\begin{array}{l}\text { Mujeres se quedan en casa y cuidan a los hijos y hom- } \\
\text { bres proveen sustento para la familia. }\end{array}$ \\
\hline $\begin{array}{l}\text { Copiar las buenas costumbres de otras personas al- } \\
\text { rededor del mundo. }\end{array}$ & $\begin{array}{l}\text { Nuestro país debe defender nuestro modo de vida en } \\
\text { vez de adoptar los modos de vida de otros países. }\end{array}$ \\
\hline $\begin{array}{l}\text { Proteger el medio ambiente así se reduzca el creci- } \\
\text { miento económico. }\end{array}$ & $\begin{array}{l}\text { Promover el crecimiento económico, aunque dañe el } \\
\text { medio ambiente. }\end{array}$ \\
\hline $\begin{array}{l}\text { Lo mejor para una sociedad es que los distintos gru- } \\
\text { pos raciales y étnicos mantengan sus propias cos- } \\
\text { tumbres y tradiciones. }\end{array}$ & $\begin{array}{l}\text { Lo mejor es que los grupos étnicos se adapten e inte- } \\
\text { gren a la sociedad en su conjunto. }\end{array}$ \\
\hline $\begin{array}{l}\text { Los inmigrantes son bienvenidos y debemos permi- } \\
\text { tir que más entren al país. }\end{array}$ & $\begin{array}{l}\text { Los inmigrantes no son bienvenidos en este país y el } \\
\text { mayor número posible debe ser devuelto al lugar } \\
\text { donde venía. }\end{array}$ \\
\hline $\begin{array}{l}\text { Aumentar la participación ciudadana en la toma de } \\
\text { decisiones. }\end{array}$ & $\begin{array}{l}\text { El gobierno toma las decisiones basado en el conoci- } \\
\text { miento de expertos. }\end{array}$ \\
\hline $\begin{array}{l}\text { El conflicto debe ser evitado a toda costa en nuestra } \\
\text { sociedad. }\end{array}$ & El conflicto es una parte normal de nuestra sociedad. \\
\hline $\begin{array}{l}\text { El libre comercio internacional con otros países nos } \\
\text { hace una nación más prospera. }\end{array}$ & $\begin{array}{l}\text { Debemos limitar el comercio internacional con otros } \\
\text { países para proteger nuestros empleos. }\end{array}$ \\
\hline
\end{tabular}

Fuente: Encuesta de comunicación política Colombia 2018 - Universidad Externado de Colombia, Universidad de Wisconsin y DeProyectos S. A. S. 


\section{Resultados}

Polarización Ideológica. De las 11 preguntas temáticas sobre la escala unidimensional liberal-conservador, se encontró que en ocho preguntas relacionadas con los temas de igualdad, rol de la familia, costumbres, economía, cultura, inmigración, participación y conflicto, alrededor del 39\% de las respuestas se ubicaron en la posición 1, asociada a la postura más liberal siendo este el porcentaje mayoritario.

Las preguntas acerca de religión y libertad civiles obtuvieron un porcentaje mayor en la posición 10 con una cifra del $31 \%$. Sin embargo, considerando que estas preguntas tienen un direccionamiento ideológico invertido con relación a las otras preguntas, las respuestas demuestran una tendencia liberal. La última pregunta de este grupo, que abarca el concepto del conflicto, obtuvo un porcentaje de 22,7\% y 22,5\% en las posiciones 1 y 10, respectivamente, demostrando un resultado similar entre los valores extremos. El valor 5, próximo al valor intermedio de la escala $(5,5)$ obtuvo el $15,7 \%$ de las respuestas, siendo el tercer valor con mayor frecuencia. El 34,3\% del promedio de las respuestas del conjunto de las preguntas se ubicaron en el valor 1, siendo este la representación de la posición más liberal en la escala. El valor 5 y 10 representaron un porcentaje de $15 \%$ y 18,4\%, respectivamente.

Expresiones políticas online. Con el propósito de conocer si la gente se expresa políticamente en redes sociales como Facebook y Twitter, se preguntó sobre la frecuencia de estas expresiones teniendo en cuenta una escala de o a 5, siendo o nunca y 5 frecuentemente. Para el caso de Facebook, el 21,9\% de los individuos se ubicó en la posición 3, el 19,2\% de encuestados indicó nunca expresarse por esta red, porcentaje similar a las personas que indicaron expresarse frecuentemente (19\%). En Twitter, el 23,9\% de los encuestados se ubicaron en la posición 4, entendiendo que la posición 5 representa expresarse frecuentemente. El 19,9\% de las personas indicaron que nunca se expresan. Por su parte, el 13,1\% señalaron expresarse frecuentemente.

Al promediar los resultados de la frecuencia de expresión política en Facebook y en Twitter, se encuentra lo siguiente: el 16,6\% de las personas se expresan frecuentemente en sus redes sociales, frente al 19\% que señalan nunca expresarse. Cerca del 28\% se ubican en el punto medio de esta escala.

Tamaño de las redes sociales. En el caso del número de amigos en Facebook, 809 personas de 1118 indicaron usar esta red. Dos personas respondieron tener únicamente un amigo en Facebook, siendo este el número menor de las respuestas obtenidas. Dos personas indicaron tener 5000 amigos en Facebook, máximo número de amigos que respondieron los encuestados. El número de amigos con mayor frecuencia fue 200 (8,2\%), seguido por 999 (6,8\%). Frente a Twitter, es necesario precisar que el número de personas que usan esta red se reduce en comparación con Facebook, en tanto 175 personas indicaron disponer de esta. El 11,4\% de las personas respondieron seguir 200 usuarios de Twitter, el cual fue el número con mayor frecuencia, seguido por 50 contactos seguidos representando el $8 \%$ de los encuestados. 
El número de amigos o contactos en Facebook y el número de personas que siguen en Twitter los encuestados fueron promediados con el propósito de identificar el tamaño de la red de las personas. Los encuestados señalaron que el número de contactos en sus redes oscilan entre 2 y 5000 , siendo 200 contacto (6.8\%) el número con mayor frecuencia.

Correlaciones, regresiones y variables mediadoras. La variable polarización ideológica presentó una correlación positiva, muy débil y significativa con expresiones políticas online, $r(815)=.101$, $p=.004$. La correlación entre polarización ideológica y tamaño de la red fue negativa, muy débil y significativa, $r(816)=-.078, p=.025$, es decir que, con el aumento de la polarización ideológica, disminuyó el tamaño de la red en la esfera digital de las personas. En el caso de expresiones políticas online, la correlación con el tamaño de las redes sociales fue positiva, muy débil y significativa, $r(818)$ $=.162, p<.001$ (Ver Tabla 2).

Tabla 2. Correlaciones de las variables polarización ideológica, expresiones políticas online y tamaño de la red.

\begin{tabular}{lccc}
\hline & 1 & 2 & 3 \\
\hline Polarización ideológica & - & $.101^{* *}$ & $-.078^{*}$ \\
Expresiones políticas online & & - & $.162^{* * *}$ \\
Tamaño de las redes sociales & & & -
\end{tabular}

${ }^{*} p<.05 ;{ }^{* *} p<.01 ;{ }^{* * *} p<.001$

Una vez efectuado el análisis de la regresión lineal simple (Ver Tabla 3), se puede afirmar que algunas variables de control presentaron una relación significativa con la variable dependiente (tamaño de la red). El tercer modelo de la regresión explicó en un $14 \%\left(R^{2}=.147\right)$ la influencia de las variables de control tales como educación, edad, género, interés en política o consumo de medios y las variables independientes (polarización ideológica y las expresiones políticas online) sobre el tamaño de la red. En este modelo, las variables de control como género ( $\beta=-.077, p=.020)$ y edad ( $\beta$ $=-.311, p<.001)$ presentaron una relación significativa con el tamaño de las redes sociales. Sin embargo, las variables de control no afectan la influencia de la polarización ideológica sobre el tamaño de la red.

Para determinar el papel mediador de las expresiones políticas online sobre la relación entre polarización ideológica y tamaño de las redes sociales, se utilizó la macro PROCESS para SPSS que permitió encontrar que el efecto indirecto de la polarización ideológica sobre el tamaño de las redes sociales es estadísticamente significativo, en tanto el intervalo de confianza no incluye el valor o $(B=$ $6.88, E E=2.88,95 \%$ IC $[2.00,13.26])$. Teniendo en cuenta que el efecto es positivo, la polarización ideológica a través de las expresiones políticas online tiende a ampliar el tamaño de las redes sociales de las personas en las plataformas digitales (Ver Figura 1). 
Tabla 3. Modelo explicativo del tamaño de la red.

\begin{tabular}{|c|c|c|c|c|c|c|}
\hline & Modelo & $B$ & $E E$ & $\beta$ & $t$ & $p$ \\
\hline \multirow[t]{4}{*}{1} & Constante & 718.479 & 72.902 & & 9.855 & .000 \\
\hline & Género & -62.507 & 27.070 & -.077 & -2.309 & .021 \\
\hline & Edad & -9.981 & 1.047 & .318 & -9.531 & .000 \\
\hline & Educación & 11.010 & 5.697 & .064 & 1.932 & .054 \\
\hline \multirow[t]{6}{*}{2} & Constante & 698.206 & 78.316 & & 8.915 & .000 \\
\hline & Género & -66.206 & 26.709 & -.082 & -2.479 & .013 \\
\hline & Edad & -9.597 & 1.036 & -.306 & -9.267 & .000 \\
\hline & Educación & 8.805 & 5.636 & .052 & 1.562 & 119 \\
\hline & $\begin{array}{l}\text { Expresiones políti- } \\
\text { cas online }\end{array}$ & 34.864 & 7.818 & .148 & 4.459 & .000 \\
\hline & $\begin{array}{l}\text { Polarización ideo- } \\
\text { lógica }\end{array}$ & -33.879 & 13.101 & -.085 & -2.586 & .010 \\
\hline \multirow[t]{8}{*}{3} & Constante & 658.134 & 81.957 & & 8.030 & .000 \\
\hline & Género & -62.744 & 26.885 & -.077 & -2.334 & .020 \\
\hline & Edad & -9.738 & 1.038 & -.311 & -9.385 & .000 \\
\hline & Educación & 6.615 & 5.802 & .039 & 1.140 & .255 \\
\hline & $\begin{array}{l}\text { Expresiones políti- } \\
\text { cas online }\end{array}$ & 32.699 & 7.970 & .139 & 4.103 & .000 \\
\hline & $\begin{array}{l}\text { Polarización ideo- } \\
\text { lógica }\end{array}$ & -33.451 & 13.113 & -.084 & -2.551 & .011 \\
\hline & Interés en política & 2.062 & 15.041 & .005 & .137 & .891 \\
\hline & $\begin{array}{l}\text { Consumo de me- } \\
\text { dios }\end{array}$ & 22.416 & 12.714 & .061 & 1.763 & .078 \\
\hline
\end{tabular}

Figura 1. Modelo de mediación simple.

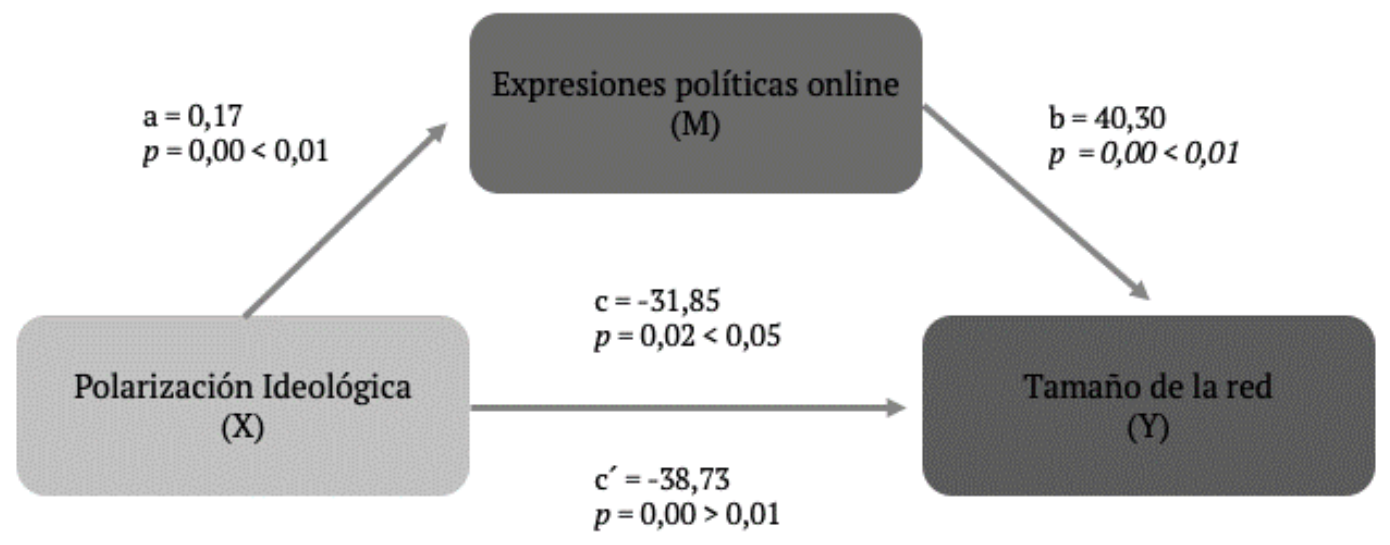

$B=6.88, S E=2.88,95 \% C I[2.00,13.26]$

Nota: $\mathrm{SE}$ = Error Estándar por las siglas en inglés; CI = Intervalo de Confianza por las siglas en inglés. 


\section{Conclusiones}

Los resultados permiten afirmar que, a partir de una asociación directa, la polarización ideológica está negativamente relacionada con el tamaño de la red de las personas $\left(c^{\prime}=-38.73, p<\right.$.oo1). Esta situación permite sostener, en primer lugar, bajo la perspectiva de Lee et al. (2014), que el aumento del tamaño de las redes sociales termina por dar apertura a la compresión de ideas opuestas, induciendo así a un efecto de despolarización. En segundo lugar, si se analiza este resultado desde otras perspectivas, las personas al prevenir la exposición a ideas contradictorias generan "cámaras de eco" en sus redes sociales, es decir, disminuyen la generación de lazos con personas de ideas diferentes, lo que termina por truncar la extensión de su red. Este factor termina por aumentar la polarización (Bail et al., 2018).

Frente a la relación entre las expresiones políticas y el tamaño de la red, los resultados evidencian una asociación positiva entre estas $(b=40.30, p<.001)$. Lo anterior podría entenderse en tanto a medida que se incrementa el flujo de información y la posibilidad de expresión pública en las redes sociales, se incrementa el número de redes interpersonales (Kavanaugh et al. 2005). Si las personas se encuentran en capacidad de comunicarse con individuos de ideas contrarias, la red será más heterogénea y se dará apertura a la generación de lazos fuertes y débiles, extendiendo el número de contactos en su red (Gil de Zúñiga \& Valenzuela, 2011).

Al testear el rol mediador de las expresiones políticas online, fue posible confirmar la hipótesis planteada: las personas que se ubican en un mayor nivel de polarización ideológica, al realizar expresiones políticas online, presentan un mayor tamaño de sus redes en plataformas digitales $(B=$ $6.88, E E=2.88,95 \%$ IC $[2.00,13.26])$. Las personas que se encuentran polarizadas buscan información de ideas contradictorias respecto a sus creencias preexistentes dado que se sienten capaces para contra argumentar esa información. En la búsqueda de ideas opuestas y en la generación de discusiones con otras personas, generan lazos débiles y conexiones que amplían el número de individuos en su red (Lee et al., 2014).

Se podría analizar en futuras investigaciones si hay una tendencia persuasiva entre las personas más polarizadas. Barnidge et al. (2018) señalan que las expresiones políticas pueden ser un intento de persuasión. Se pensaría que aquellas personas que se encuentran más polarizadas buscan cambiar las opiniones de los demás hacia sus ideas, de ahí que se propongan ampliar su red generando mayores conexiones con otros individuos para aumentar así su capacidad de persuasión. El estudio adelantado permite pensar que las redes sociales están dando apertura a procesos deliberativos al ser escenarios que facilitan la exposición de las personas a diferencias políticas, es decir, se constituyen como un nuevo espacio para la deliberación pública. A pesar de que sea un espacio propicio para el aumento de la polarización ideológica, las redes sociales permiten que, a partir de la deliberación, las personas puedan pensar críticamente sobre las diferentes perspectivas de un asunto público (Rengifo, 2019). 
Una vez concluida la investigación, se observa la necesidad de investigar los diferentes fenómenos políticos a la luz del modelo de mediación comunicativa (Shah et al., 2017). Para superar las limitaciones metodológicas de este estudio, se debe realizar un análisis moderador de otras variables que se desprendan del contexto y adoptar un enfoque longitudinal que permita afirmar o no la permanencia de la mediación. Se entiende que los resultados del presente estudio no permiten hacer generalidades en el tiempo. Con relación al alcance de este estudio, hace falta una comparación del tamaño de las redes sociales de personas con ideas más liberales en oposición al tamaño de las redes de las personas con ideas más conservadoras, representando una oportunidad para futuras investigaciones. En un escenario de conflictos que afectan a la sociedad colombiana, la comunicación política puede sugerir alternativas para fortalecer la deliberación en el ejercicio de la política.

\section{Agradecimientos}

Agradecemos el apoyo brindado por parte de la Universidad Externado de Colombia, en especial a Diego Mazorra, director académico de la Maestría en Comunicación Política y a la profesora Angela Duarte por sus observaciones a lo largo del ejercicio investigativo. Este artículo se desprende de la investigación realizada como requisito para obtener el título de la maestría en comunicación política de la Universidad Externado de Colombia por parte de sus autores.

\section{Declaración de conflicto de intereses}

Los autores no informaron ningún posible conflicto de intereses.

\section{Referencias}

Abramowitz, A. (2010). The disappearing center: Engaged citizens, polarization, and American democracy. Yale University Press.

Alconchel. G. (2014). Impacto de las redes sociales sobre el debate político. Anduli, 13, 29-42.

Ardèvol-Abreu, A., Barnidge, M., \& Gil de Zúñiga, H. (2017). Communicative antecedents of political persuasion: Political discussion, citizen news creation, and the moderating role of strength of partisanship. Mass Communication and Society, 20(2), 169-91. https://doi.org/10.1080/15205436.2016.1244855

Bail, C.A., Argyle, L.P., Brown, T.W., Bumpus, J.P., Chen, H., Hunzaker, M. B. F., Lee, J. Mann, M., Merhout, F., \& Volfovsky, A. (2018). Exposure to opposing views on social media can increase political polarization. PNAS, 115(37), 9216-9221. https://doi.org/10.1073/pnas.1804840115

Barnidge, M., Huber, B., Gil de Zúñiga, H., \& Liu, J. H. (2018). Social media as a sphere for "risky" political expression: A twenty-country multilevel comparative analysis. The International Journal of Press/Politics, 23(2), 161-182. https://doi.org/10.1177/1940161218773838 
Brundidge, J. (2010). Encountering "difference" in the contemporary public sphere: The contribution of the internet to the heterogeneity of political discussion networks. Journal of Communication, 6o(4), 680-700. https://doi.org/10.1111/j.1460-2466.2010.01509.x

Campbell, J. (2016). Polarized: Making sense of a divided America. Princeton University Press.

Delli Carpini, M. X. D., \& Keeter, S. (1993). Measuring political knowledge: Putting first things first. American Journal of Political Science, 37, 1179-1206. https://doi.org/10.2307/2111549

Domínguez. H. (2013). Democracia deliberativa en Jürgen Habermas. Analecta Política, 4(5), 302326.

Dueñas, M. (2019, 8 de agosto). Duque llama a superar la polarización en Colombia al cumplir un año de gobierno. Agencia EFE. Recuperado de https://www.efe.com/efe/america/politica/duquellama-a-superar-la-polarizacion-en-colombia-al-cumplir-un-ano-de-gobierno/200000354039376

Eveland, W. P., Hutchens, M. J., \& Morey, A. C. (2013). Political network size and its antecedents and consequences. Political Communication, 30(3), 371-394. https://doi.org/10.1080/10584609.2012.737433

Fiorina, M. P., Abrams, S. J., \& Pope, J. C. (2005). Culture war? The myth of a polarized America. Pearson Longman.

García-Guadilla, M. P., \& Mallen, A. (2019). Polarization, participatory democracy, and democratic erosion in Venezuela's twenty-first century socialism. The ANNALS of the American Academy of Political and Social Science, 681(1), 62-77. https://doi.org/10.1177/0002716218817733

Gil de Zúñiga, H., \& Valenzuela, S. (2011). The mediating path to a stronger citizenship: Online and offline networks, weak ties, and civic engagement. Communication Research, 38(3), 397-421. https://doi.org/10.1177/0093650210384984

Goode, L. (2009). Social news, citizen journalism and democracy. New Media \& Society, 11(8), 12871305. https://doi.org/10.1177/1461444809341393

Hayes, A. (2017). Introduction to mediation, moderation, and conditional process analysis. A regression-based approach. Gilford Press.

Hernández Sampieri, R., Fernández Collado, C., \& Baptista Lucio, M. P. (2010). Metodología de la investigación. Mc Graw Hill.

Katsambekis, G., \& Stavrakakis, Y. (2013). Populism, anti-populism and European democracy: A view from the south. Open Democracy. Recuperado de https://www.opendemocracy.net/caneurope-make-it/giorgos-katsambekis-yannis-stavrakakis/populism-antipopulism-and-european-democr/

Kavanaugh, A., Reese, D., Carroll, J., \& Rosson, M. (2005). Weak ties in networked communities. The Information Society, 21(2), 119-131. https://doi.org/10.1080/01972240590925320

Klapper, J. T. (1960). The Effects of Mass Communication. Social Forces, 4O(1), 98-98. https://doi. org/10.2307/2573494

Lee, J.K., Choi, J., Kim, C., \& Kim, Y. (2014). Social media, network heterogeneity, and opinion polarization. Journal of communication, 64(4), 702-722. https://doi.org/10.1111/jcom.12077

Lindh, J., Fábrega, J., \& González, J. (2019). La fragilidad de los consensos. Polarización ideológica en el Chile de Pinochet. Revista de Ciencia Política, 39, 99-127. http:// doi.org/10.4067/So718-090X2019000100099 
Lozada, M., (2014). Us or them? Social representations and imaginaries of the other in Venezuela, Papers on Social Representations, 23, 21.1-21.16. Recuperado de https://pdfs.semanticscholar.org/62da/77018e16a5c67682db232c7556e1d1e93bc8.pdf?_ga=2.134618609.1232030394. $1582253885-21782413.1582253885$

McCoy, J., Rahman, T., \& Somer, M. (2018). Polarization and the global crisis of democracy: common patterns, dynamics, and pernicious consequences for democratic polities. American Behavioral Scientist, 62(1), 16-42. https://doi.org/10.1177/0002764218759576

Nip, J. (2006). Exploring the second phase of public journalism. Journalism Studies, 7(2), 212-236, https://doi.org/10.1080/14616700500533528

Oquendo. A. (2005). Más allá de la democracia deliberativa. Polis, Revista Latinoamericana, 10,139.

Park, C. S. (2017). Unpacking the Relationship between mobile phone usage patterns, network size, and civic engagement. Online Journal of Communication and Media Technologies, 7(2), 169197. https://doi.org/10.29333/ojcmt/2596

Plata, J. C. (2016). ¿Polarización? Posiciones ideológicas durante la campaña presidencial colombiana en 2014". Colombia Internacional, (87), 199-215. https://doi.org/10.7440/colombiaint87.2016.08

Poole, K., \& Rosenthal, H. (2006). Congress: A political economic history of roll call voting. Oxford University Press.

Presidencia de la República. (2018, 7 de agosto). El pacto por Colombia. Discurso de posesión del Presidente de la República, Iván Duque Márquez. Recuperado de https://id.presidencia.gov.co/Paginas/prensa/2018/El-Pacto-por-COLOMBIA-Discurso-de-Posesion-del-Presidente-de-la-Republica-Ivan-Duque-Marquez.aspx

Rengifo. C. (2019). Internet, deliberación y democracia. NOVUM, 2(9), 65-85.

Revista Dinero. (2019, 21 de marzo). El impacto de la polarización en la economía. Revista Dinero. Recuperado de https://www.dinero.com/edicion-impresa/editorial/articulo/el-impacto-dela-polarizacion-politica-en-la-economia/268561

Revista Semana. (2019, 31 de julio). Si la mayoría de los colombianos son de centro, ¿Existe la polarización? Revista Semana. Recuperado desde https://www.semana.com/nacion/articulo/existe-la-polarizacion-en-colombia/626002

Rojas, H. (2018). Tendencias y desafíos en comunicación política. (Artículo inédito). Universidad Externado de Colombia.

Rojas, H., Barnidge, M., \& Abril, E. P. (2016). Egocentric publics and corrective action. Communication and the Public, 1(1), 27-38. https://doi.org/10.1177/2057047315619421

Rojas, H., Shah, D. V., \& Friedland, L. A. (2011). A communicative approach to social capital. Journal of Communication, 61(4), 689-712. https://doi.org/10.1111/j.1460-2466.2011.01571.x

Saiegh, S. (2014). Partisanship, ideology, and representation in Latin America. (Working Paper Series $\mathrm{N}^{\circ}$ IDB-WP-533). Washington: Inter-American Development Bank.

Shah, D. V., McLeod, D. M., Rojas, H., Cho, J., Wagner, M. W., \& Friedland, L. A. (2017). Revising the communication mediation model for a new political communication ecology. Human Communication Research, 43(4), 491-504. https://doi.org/10.1111/hcre.12115

Valenzuela, S., \& Rojas, H. (2019). Taming the digital information tide to promote equality. Nature Human Behaviour 3, 1134 - 1136 https://doi.org/10.1038/s41562-019-0700-9 
Villalba, A. (2019, 11 de junio). Polarización: un debate de muchas emociones y pocos argumentos. Universidad de los Andes. Recuperado de https://uniandes.edu.co/es/noticias/sociologia/polarizacion-un-debate-de-emociones-sin-argumentos

Villalobos, J. (2017). Del miedo a la ingobernabilidad. La salvadoreñización de Colombia. FIP-Fundación ideas para la paz. Bogotá.

Yarce, J. (2018, 6 de enero). La polarización política y social. La República. Recuperado de https://www.larepublica.co/analisis/jorge-yarce-2577840/la-polarizacion-politica-y-social2586384 